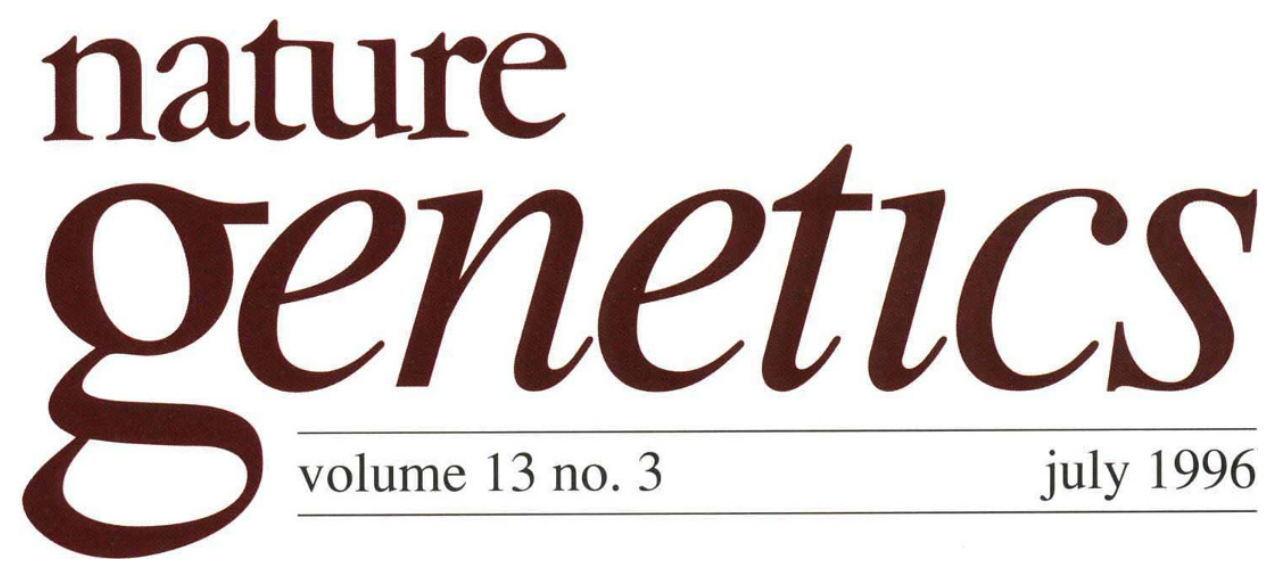

\title{
Delivering the knockout punch
}

It is difficult to pick up any reputable research journal these days and not find one or more papers describing the latest results of gene tampering in mice. These reports frequently include some variation of aberrantly behaved, memoryimpaired or developmentally deformed animal which the authors believe (and editors are frequently persuaded) will yield important advances in physiology or cell biology, or provide an unparalleled means for modelling a human genetic disease. Not every experiment produces phenotypes as dramatic as sex reversal or overtly aggressive behaviour, of course. Many genes that have been targeted perform such essential functions that the result is early prenatal death, although the causes of these fatalities are actually fairly limited, and include disturbances in the vascular circulation and defects in setting up liver-based haematopoiesis ${ }^{1}$.

With the technical problems regarding efficiency of recombination in embryonic stem cells that plagued the field a few years ago all but overcome, a torrent of intrinsically valuable information is flowing into the journals (and databases). A compendium of more than 250 mouse gene knockouts was published last year ${ }^{2-4}$, and there is now a powerful database accessible on the Internet that can be searched for information by phenotype, targeting method, and so on (TBASE, http://www.gdb.org/Dan/tbase/tbase.html). Summaries of submissions to TBASE, which already number close to 1,000 in total, have been appearing quarterly in Trends in Genetics this year ${ }^{5}$. Of course, the results are not always what one might expect - but, then again, that is one of the pleasures of doing science.

This issue of Nature Genetics contains its fair share of gene targeting reports, featuring two papers on mouse models for myotonic dystrophy, two related integrin knockouts, the characterization (and partial rescue of) mice deficient for a homeobox gene called Cart1, and a knockout of the mismatch repair gene, Mlh1. Each of these papers illustrates some of the main reasons for creating such models in the first place. And yet often the results are not as straightforward as one might expect. As discussed by Wynshaw-Boris on page 259, the long-awaited myotonic dystrophy mice, produced by targeting the DM-kinase gene, show some familiar signs of the human phenotype as they age, but are lacking in others (pages 316 and 325). There is still uncertainty as to the precise mechanism (loss or gain of function) in this unusual trinucleotide repeat disease - indeed, Wieringa and colleagues went so far as to construct knockout and transgenic mice. There is also the interesting suggestion that the CTG expansion at the 3 ' end of the kinase might disrupt the expression of one or more adjacent genes. While the mouse models may have their 


\section{editorial}

1. Copp, A.J. Trends Genet. 11, 87-93 (1995).

2. Brandon, E.P., Idzerda, R.L. \& McKnight, G.S Curr. Biol. 5, 625-634 (1995).

3. Brandon, E.P., Idzerda, R.L. \& McKnight, G.S. Cur. Biol. 5, 758-765 (1995).

4. Brandon, E.P., Idzerda, R.L. \& McKnight, G.S. Curr. Biol. 5, 873-881 (1995).

5. Anagnostopoulos, A.V. \& Jacobson, D. Trends Genet. 12, 236 \{1996)

6. Nakayama, K. et al. Cell 85, 707-720 (1996).

7. Kiyokawa, H. et al. Cell 85, 721-732 (1996).

8. Kuhn, R. et al. Science 269, 1427-1429 (1995)

9. Threadgill, D.W. ot al. Science 269, 230-234 (1995). limitations as a pure model of the human disease, these results assume greater importance in view of the difficulties in unravelling the mechanism of the disorder.

On pages 366 and 370, two European groups present the complementary results of targeting the two subunits of $\alpha 6 \beta 4$ integrin, which is an important component of hemidesmosomes - adhesion structures that are required for attachment of basal cells to the basement membrane. Sonnenberg and colleagues in Amsterdam (page 366) have generated $\beta 4$ integrin null mice, and find that the homozygotes suffer from loose, fragile, blistered skin even more severe than the corresponding human disorder, epidermolysis bullosa. Interestingly, hemidesmosomes are absent in these mice. This proves that $\beta 4$ integrin is required for the stable adhesion of keratinocytes to the underlying basement membrane. Georges-Labouesse and coworkers from Strasbourg (page 370) reveal a very similar phenotype in mice lacking the $\alpha 6$ integrin, including the complete absence of hemidesmosomes. These findings are something of a surprise, however, given that this subunit is widely expressed, and was expected to have a significant role in embryonic development (although the mice do die shortly after birth). Aside from the valuable information these mice will provide into the function of $\alpha 6$ integrin, they also highlight another candidate gene for skin blistering disorders in humans.

One of the main areas for gene targeting is, of course, to examine the effect of specific genes in mouse embryonic development. Such is the rationale for the study from de Crombrugghe and colleagues on page 275. They targeted the Cart1 homeobox gene, which encodes a transcription factor, cartilage homeoprotein 1. Homozygous animals die within 24 hours of birth suffering from acrania due to lack of neural tube closure at the forebrain/midbrain boundary. The researchers also examined the effect on neural tube formation of treating Cartl mutants with large doses of folic acid during pregnancy. Thirteen out of 16 mice developed fully formed craniums, and five appeared normal (although they all died within a couple of days). Whether, as the authors suggest, the folic acid alters the head mesenchyme to facilitate closure at the forebrain-midbrain boundary remains to be seen, but their study should stimulate other workers to examine the potential role of folate in mouse models.

Another popular class of genes for knockout experiments are genes involved in cancer. On page 336 of this issue, Liskay, Ashley and colleagues have targeted the Mlh1 mismatch repair gene. In keeping with studies on related genes, homozygous mice show microsatellite instability; however, both sexes are infertile. Abnormalities in gametogenesis were confirmed in antibody studies, which revealed a striking pattern of MLH1 localization at sites of chiasma formation during meiosis (see also the News \& Views on page 261).

Many mouse mutants succumb so early in embryonic development that it is difficult to conclude much about the gene's function other than its requirement for viability. On the other hand, some investigators set out expecting to see a fatal phenotype, only to be (pleasantly) surprised. Such was the case in two recent reports $^{6,7}$ of mice lacking the p27 $7^{\text {Kipl }}$ gene, which codes for an inhibitor of cyclin/cyclin-dependant kinases. Rather than observing embryos with irregular tissue organization, the mice were healthy and grew significantly larger than wildtype after about a month. Later on, the mice developed pituitary tumours, similar to the unexpected finding in mice lacking the retinoblastoma gene homologue, and suggesting a genetic link between the two.

The mouse knockout field shows no signs of abating, particularly as ever more malleable techniques for the timing and specificity of the targeting events come into play $^{8}$. The effects of creating double mutants, varying the targeting construct (compare the variations in behavioural phenotypes of mouse models for Huntington's disease for example) and altering the background strain ${ }^{9}$ are also important parameters that will feature more heavily in the future. As the pace of gene discovery speeds up, the knockout punches will be hard to fend off. 